\title{
The electrochemical oxidation of sulphite on gold electrodes
}

\author{
Xiaosheng Cai, ${ }^{\mathrm{a}}$ Chuhong Lin, ${ }^{\mathrm{b} \dagger}$ John S. Foord, ${ }^{\mathrm{a}}$ Richard G. Compton ${ }^{\mathrm{b} *}$ \\ a Department of Chemistry, Chemistry Research Laboratory, University of Oxford, Mansfield Road, Oxford OX1 3TA, United \\ Kingdom \\ b Department of Chemistry, Physical and Theoretical Chemistry Laboratory, University of Oxford, South Parks Road, Oxford \\ OX1 3QZ, United Kingdom \\ * e-mail: richard.compton@chem.ox.ac.uk
}

Received: ((will be filled in by the editorial staff))

Accepted: ((will be filled in by the editorial staff))

\begin{abstract}
The electro-oxidation of sulphite is studied in acid media on gold macroelectrodes and Au particle array modified boron doped diamond electrodes. The sulphite oxidation proceeds through a one-electron transfer process followed by a chemical step of second order ( $\mathrm{EC}_{2}$ mechanism), as evidenced by experimental voltammetry and digital simulation for both gold macroelectrodes and gold particle modified substrates. The diffusion coefficient of sulphite is calculated consistently for both cases at about $4 \times 10^{-9} \mathrm{~m}^{2} \mathrm{~s}^{-1}$, with the reverse peak behaviour concentration dependent. The arrays are made by electrodeposition and show a morphological transition from a quasi-spherical to a star shape as the growth time of the particles increases. Kinetic parameters inferred from the electro-oxidation at the macroelectrode can be successfully applied to model the gold particle modified electrode data to confirm the $\mathrm{EC}_{2}$ mechanism, whilst recognising the overlapping diffusion layers of adjacent gold particles.
\end{abstract}

Keywords: gold macroelectrode, gold particle modified boron doped diamond, sulphite electro-oxidation, voltammetry

DOI: 10.1002/elan.((will be filled in by the editorial staff))

\section{Introduction}

The electrochemical oxidation of aqueous sulphite solutions has attracted attention because of the wide application of sulphite in industry as preservatives in foods like alcoholic beverages (such as beer and wine), soft drinks, and dried fruits [1]. However levels need to be controlled since excessive exposure may have harmful effects such as gastrointestinal problems and respiratory disorders [2]. Hence, its determination is of much importance and interest [3-4]. To provide a platform for robust electroanalytical methods, it is vital to decipher the electrochemical process in terms of the mechanism of oxidation and reduction, which has been a focus of earlier investigations but remains an open question [5-17].

The electrochemical reactivity of the sulphite system has been studied in different electrolyte media and at various electrode materials. In particular carbon has been utilised by electrochemists in many forms including activated carbon, graphite, glassy carbon, and carbon black [18-23]. However, the electro-oxidation of sulphite at carbon electrodes usually requires a high overpotential and is a kinetically sluggish process [21].
Hence attention has been paid to metals, which may decrease the overpotential and show catalytic ability towards the sought reaction [24].

Metal oxides including $\mathrm{PbO}_{2}$ [25-26] have been studied as well as noble metals, such as Pd [27-28], Pt $[5-11,16]$ and Au [11-16], which are recognized as effective electrocatalysts. For the achievement of high reactivity efficiency and the saving of the metal volume, however, construction of nanostructures in the form of small particles deposited on various substrates such as ceramics or boron-doped diamond (BDD) has been utilised [29-30]. Note that BDD electrodes are widely used wafers for electrochemical applications in terms of their wide potential window, low background current, mechanical robustness, good chemical stability and resistance to fouling [31-33]. The improved diffusion based on convergent at nanoparticles can provide a higher local mass transport rate to the electrode surface provided adjacent diffusion fields do not overlap [34]. Meanwhile high effective surface areas and interfacedominated properties can be obtained at the nanoscale along with many active sites and a better signal-to-noise 
ratio [35]. Furthermore, the excellent electrocatalytic properties of some nanometer-sized particles can endow greater reversibility for redox processes making the processes more kinetically viable compared to the same electrode materials in bulk [36-37], and the effect of nanoparticle size in electrocatalytic reaction has been reported [38-39]. Last but not least, the local microenvironment can be controlled via the use of nanoparticles for biomolecule immobilization [35, 40].

In terms of the mechanistic aspects of the electrooxidation of sulphite, there have been numerous welldocumented studies using bulk Pt [6-10] as a preferred electrocatalytic electrode but relatively fewer on bulk Au. The latter is the focus of this paper. Several mechanisms have been proposed for the sulphite oxidation. Seo and Sawyer [11] studied the oxidation of sulphite on bulk gold in acidic solutions by means of voltammetric and chronopotentiometric techniques and observed two oxidative processes where the first one was a pure electrochemical charge transfer process while the second one was a chemical reaction between electrochemically generated metal oxide and the sulphite species. Moreover, a two-electron oxidation for the overall reaction of an irreversible nature was indicated. Samec and Weber [12] provided a further insight into the electrochemical behaviour of sulphite on gold macroelectrodes in acidic media, inferring the involvement of "strongly" or "weakly" adsorbed hydrated sulphur dioxide, and a S(VI) intermediate species which reacted with water to form the final product of bisulphate or sulphate. That said, other work [13] using voltammetry and radiotracer studies indicated the formation of both bisulphate/sulphate ( $\left.\mathrm{HSO}_{4}{ }^{-} / \mathrm{SO}_{4}{ }^{2-}\right)$ and dithionate $\left(\mathrm{S}_{2} \mathrm{O}_{6}{ }^{2-}\right)$ upon electrooxidation of aqueous sulphite solutions on bulk gold. These findings may be conflicting, which reflects the intrinsic complexity of electrochemistry of sulphite in terms of its reactivity and various valence states, and the well-grounded dependence on the corresponding electrochemical context. On the other hand, mechanistic observations of aqueous sulphite oxidation on wafer supported disperse catalysts to compare the behaviour to its bulk metal electrode established decades of years ago are scarce [41-43]. In the light of the reported better intrinsic performance of gold compared with platinum towards sulphite oxidation allied to its much stronger absorption on platinum [14], the advantages of nanoparticles, and the antecedents mentioned before, in this article we report new findings acquired by cyclic voltammetry (CV) studies of this process using gold particle arrays deposited on BDD in acid media coupled with digital simulation, and in particular offer insight into mechanistic aspects of sulphite oxidation. Importantly, it is shown that the mechanism of sulphite oxidation on gold can be elucidated with simulation of data acquired at Au particle arrays supported on a BDD electrode, whereas for a bulk gold electrode the greater capacitance precludes quantitative analysis.

\section{Experimental}

\subsection{Chemical reagents}

Hydrochloric acid (analytical reagent grade, 37\%), nitric acid (analytical reagent grade, 70\%), and sodium sulphite anhydrous (laboratory reagent grade) were purchased from Fisher Chemical. Sulphuric acid (ACS reagent, 95.097.0\%) and gold chloride trihydrate $(\geq 99.9 \%)$ were obtained from Sigma-Aldrich. Aqua regia was prepared in a volume ratio of $1: 3$ between $\mathrm{HNO}_{3}$ and $\mathrm{HCl}$. All the chemicals were used as received without further purification. All aqueous solutions were made with using ultrapure water from Millipore with a resistivity of close to $18.2 \mathrm{M} \Omega \mathrm{cm}$ at $25^{\circ} \mathrm{C}$.

\subsection{Electrochemical apparatus and experiment}

All the electrochemical measurements were undertaken using a computer controlled Autolab PGSTAT128N potentiostat (Metrohm Autolab, Utrecht, Netherlands) equipped with Nova software. A standard three-electrode system was employed for all electrochemical measurements. A gold macroelectrode of $2.0 \mathrm{~mm}$ diameter (CH Instruments, USA) and a boron doped diamond wafer ( $\mathrm{B} \approx 5 \times 10^{20}$ atoms $\mathrm{cm}^{-3}$, Element Six) were used as working electrodes. BDD was embedded in a home built polytetrafluoroethylene electrochemical cell with an exposed area of $0.37 \mathrm{~cm}^{2}$ to the electrolyte, which has been described before [44]. A silver/silver chloride electrode $(1.0 \mathrm{M} \mathrm{KCl})$ was used as a reference electrode and a platinum coil served as a counter electrode. All the potentials reported in this work were with respect to this reference electrode, unless specified.

Prior to use, the BDD electrode was cleaned in a vial containing aqua regia overnight to remove the previous gold residuals between experiments and immersed in $0.10 \mathrm{M} \mathrm{HNO}_{3}$ for voltammetric cycles between -1.7 to $2.5 \mathrm{~V}$ at $0.1 \mathrm{~V} \mathrm{~s}^{-1}$. Before use, the gold electrode was polished sequentially on lapping pads with aqueous slurries of $1 \mu \mathrm{m}, 0.3 \mu \mathrm{m}, 0.05 \mu \mathrm{m}$ alumina powder (Buehler, USA), followed by rinsing and sonicating with water to remove the slurry residues.

The electrochemical deposition of gold on the BDD surface was undertaken in $0.10 \mathrm{M} \mathrm{H}_{2} \mathrm{SO}_{4}$ containing 1.0 
$\mathrm{mM} \mathrm{HAuCl}_{4}$ according to the literature [45-46]. The method included one nucleation-growing pulse. The nucleation pulse was applied at $-0.7 \mathrm{~V}$ for $2 \mathrm{~s}$, followed by growth pulses at $0 \mathrm{~V}$ for different times of $10 \mathrm{~s}, 50 \mathrm{~s}$, or $150 \mathrm{~s}$ to form $\mathrm{Au}$ particles herein referred to as $\mathrm{Au}$ (10), Au (50), Au (150), respectively. The particles without the growing stage were studied for comparison, and were labelled as $\mathrm{Au}(0)$.

The sulphite measurements were carried out by cyclic voltammetry in $0.10 \mathrm{M} \mathrm{H}_{2} \mathrm{SO}_{4}(\mathrm{pH}=0.89)$ containing different analyte concentrations of $10 \mu \mathrm{M}-2.0 \mathrm{mM}$ at different scan rates using $\mathrm{Au}$ particle modified BDDs and Au macroelectrodes.

\subsection{Characterisation}

The gold particles were characterised using Scanning Electron Microscope (SEM, Hitachi S-4300, Japan) with an acceleration voltage of $10 \mathrm{kV}$. Microscopy images were analysed for particle size and separation using ImageJ (National Institute of Health, USA). The bar in the SEM images was used for scale calibration. The "Analyze Particles" function from the "Analyze" toolbox was used to count the number of particles and measure the size. The areas of interest were selected and marked "by hand" via the user interface, and the "Measure" function from the "Analyze" toolkit was utilised to determine the chosen areas.

\subsection{Simulation}

The simulation program DigiSim (Version 3.03b, Bioanalytical Systems, Inc., West Lafayette, IN, USA) developed by Rudolph [47] was employed to model the cyclic voltammograms for sulphite oxidation. A simplified reaction mechanism was entered into the simulator:

$$
\mathrm{A} \stackrel{E^{0}, \alpha, k_{S}}{\longrightarrow} \mathrm{B}+\mathrm{e}
$$

$\mathrm{B}+\mathrm{B} \stackrel{K_{e q}, k_{f}, k_{b}}{\longrightarrow} \mathrm{C}$

where $\mathrm{A}$ is the sulphite, $\mathrm{B}$ is a reaction intermediate, and $\mathrm{C}$ is a dimer. The following parameters were used: $E^{0}$ is the formal potential, $\alpha$ is the charge transfer coefficient, and $k_{s}$ is the heterogeneous electron transfer rate constant with the Butler-Volmer model assumed to apply; $k_{f}$ and $k_{b}$ are the forward (second order) and backward (first order) rate constants of the chemical step, respectively, with their ratio corresponding to the equilibrium constant $K_{e q}$. Semiinfinite linear diffusion system was assumed and preequilibration was enabled for all the fittings.

\section{Results and Discussion}

\subsection{Electro-oxidation of sulphite at a gold macroelectrode}

The focus of this work is the oxidation at gold electrodes of sulphite in aqueous solution. Initially the voltammetric response was studied using a gold macroelectrode of 2.0 $\mathrm{mm}$ diameter. Fig. 1 shows cyclic voltammograms of 1.0 $\mathrm{mM} \mathrm{Na}_{2} \mathrm{SO}_{3}$ in $0.10 \mathrm{M} \mathrm{H}_{2} \mathrm{SO}_{4}$ at different scan rates. It is clear that all CV curves have well-defined anodic peaks. The peak potentials of sulphite oxidation lie between 0.4 and $0.5 \mathrm{~V}$ vs. Ag/AgCl. This feature is consistent with the reported work earlier in terms of shape and potential [16]. The peak potential shifts negatively with the decrease of scan rate. This will be discussed later in this paper. The plot of peak current of sulphite oxidation versus the square root of scan rate is linear, indicating that the electrochemical oxidation of sulphite is diffusion controlled. The slope $\left(5.2 \times 10^{-5} \mathrm{~A} \mathrm{~V}^{-0.5} \mathrm{~s}^{0.5}\right)$ of the plot is used to calculate the diffusion coefficient of sulphite, yielding the values of $3.8 \times 10^{-9} \mathrm{~m}^{2} \mathrm{~s}^{-1}$ and $4.8 \times 10^{-10} \mathrm{~m}^{2}$ $\mathrm{s}^{-1}$, respectively, based on one- and two-electron processes, according to the Randles-Sevcik equation for an electrochemically reversible reaction (discussed later):

$I_{p}=2.69 \times 10^{5} A D^{0.5} C v^{0.5} n^{1.5}$

where $I_{p}$ is the peak current, $A$ is the electrode area, $D$ is the diffusion coefficient of reactant species, $C$ is the bulk concentration of species, $v$ is the scan rate, $n$ is the total number of electrons. Note that a previous literature report assumed a two-electron process in contrast to the conclusion below [11].

The voltammograms were further analysed in order to characterise the electron transfer process. As shown in Fig. S1, the Tafel analysis region were taken from the initial part of the ascending profile of the anodic peak. From the forward scan a plot of $\ln I$ versus $E$ was drawn with the linear fitting shown in the inset. The slope of the resulting plot was used to calculate the anodic transfer coefficient $(\beta)$ via the equation slope $=\frac{\beta F}{R T}$ where $F$ is the Faraday constant, $R$ is the universal gas constant, and $T$ is the temperature [48]. The calculated value of transfer coefficient was $0.88 \pm 0.09$ for the range of scan rates in Table S1. This apparent value is close to unity, indicating that the process is seemingly likely a one-electron transfer reversible reaction at first glance [49]. However, the positive shift of potential with the increase of scan rate suggests a more complex process since for a simple pure reversible reaction the peak potential would be independent of scan rate [50]. 

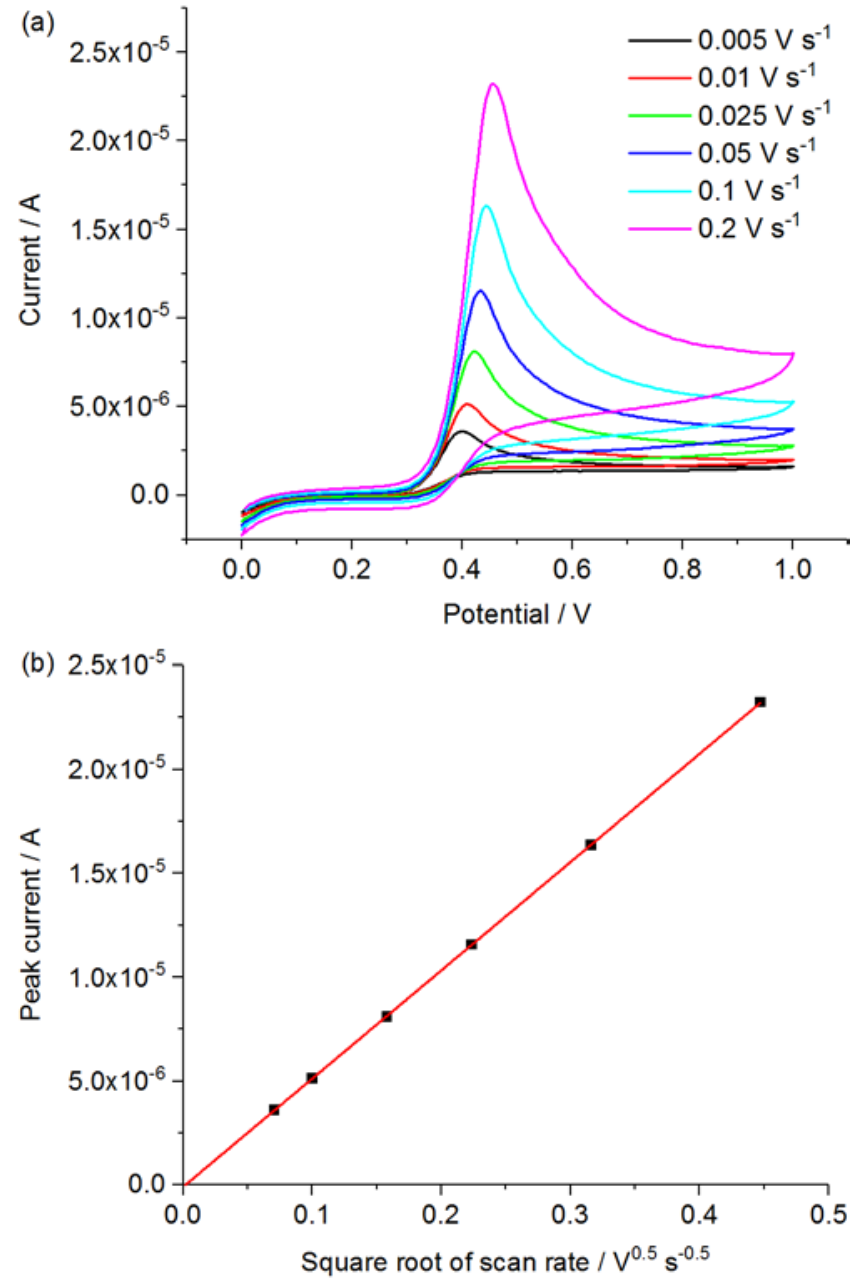

Fig. 1. (a) $\mathrm{CV}$ of Au macroelectrode in $0.10 \mathrm{M} \mathrm{H}_{2} \mathrm{SO}_{4}$ containing $1.0 \mathrm{mM} \mathrm{Na}_{2} \mathrm{SO}_{3}$ recorded at different scan rates. (b) Plot of peak current as a function of the square root of scan rate from 0.005 to $0.2 \mathrm{~V} \mathrm{~s}^{-1}$.

The electro-oxidation of sulphite was then studied at different concentrations of 10,100 , and $1000 \mu \mathrm{M}$. The CVs of sulphite oxidation at these three concentrations measured using a gold macroelectrode are shown at a scan rate of $0.05 \mathrm{~V} \mathrm{~s}^{-1}$ in Fig. S2. To enable observation of the changing voltammogram shape, the currents were normalized to the oxidation peak current. From Fig. S2 it is noteworthy that as the concentration decreases to $10 \mu \mathrm{M}$, a backward peak appears, and the wave moves to more positive potential. This likely suggests a change of concentration affecting the mechanism. We focus on the data obtained at $1.0 \mathrm{mM}$ concentration which has good signal background characteristics. Given that the Tafel analysis above is consistent with an electrochemically reversible oxidation the observed behaviour could be due to a following chemical reaction in particular [51]. The idea of an EC type scheme was considered first, where E represents the electrochemical reaction and $\mathrm{C}$ denotes a first order chemical reaction using the Testa and Reinmuth notation [52]. The lack of any back peak in the $1.0 \mathrm{mM}$ data is consistent with a fast following reaction and, in the case of a reversible electron transfer, the shift of the oxidation wave with potential. On the other hand, the emergence of a back peak in the lower concentration data suggests that the homogeneous kinetics are likely not first order. Therefore alternatively an $\mathrm{EC}_{2}$ (the electrochemical reaction followed by a chemical step of second order) mechanism can be proposed [53], since the presence of a back peak depends on both the scan rate and the concentration of the analyte in an $\mathrm{EC}_{2}$ process. We next simulated this hypothesis and the scan rate dependence of the $1.0 \mathrm{mM}$ data using DigiSim software described in the simulation section using the following scheme.

$$
\begin{aligned}
& \mathrm{A} \stackrel{E^{0}, \alpha, k_{s}}{\longrightarrow} \mathrm{B}+\mathrm{e} \\
& \mathrm{B}+\mathrm{B} \stackrel{K_{e q}, k_{f}, k_{b}}{\longrightarrow} \mathrm{C}
\end{aligned}
$$

The parameters are given as following: $E^{0}=0.4 \mathrm{~V}, \alpha=$ $0.5, k_{s}=0.01 \mathrm{~cm} \mathrm{~s}^{-1}, K_{e q}=1 \times 10^{6}, k_{f}=1 \times 10^{4} \mathrm{M}^{-1} \mathrm{~s}^{-1}, D_{A}$ $=D_{B}=D_{C}=4 \times 10^{-9} \mathrm{~m}^{2} \mathrm{~s}^{-1}$, working electrode area $A=$ $3.14 \times 10^{-6} \mathrm{~m}^{2}$. In order to optimize the fit across various voltage scan rates, the transfer coefficient was assumed to be 0.5 which with a fast $k_{s}$ value would give reversible behaviour, and the diffusion coefficients of the species were presumed to be equal. The simulated diffusion coefficient of electroactive species is close to the calculated experiment value above on the basis of the one electron transfer assumption. Fig. 2 shows the overlaid experimental and simulated voltammograms at different scan rates of from 0.005 to $0.2 \mathrm{~V} \mathrm{~s}^{-1}$. There is a good fit across the full range of scan rates in terms of voltammogram shapes as well as peak currents and peak potentials. The mismatches in the reverse scan reflect the background capacitance but capture exactly the lack of a back peak. The presence or absence of a reverse peak is dependent on a dimensionless kinetic rate constant $K_{2}=\frac{k_{f}}{v}\left(\frac{R T}{F}\right)[A]_{b u l k}$, which is proportional to the analyte concentration $\left([A]_{b u l k}\right)$ and inversely proportional to the scan rate $(v)$ for an $\mathrm{EC}_{2}$ as mentioned before [50]. From Fig. 2 at a such high concentration of $1.0 \mathrm{mM}$ sulphite the loss of a back peak occurs for large $\mathrm{K}_{2}$. Returning to Fig. S2 the data shown for $100 \mu \mathrm{M}$ shows two forward peaks and the possible emergence of a back peak. This suggests the "turn off" of the following chemical step as the product is diluted with 
(a) $4.0 \times 10^{-6}$
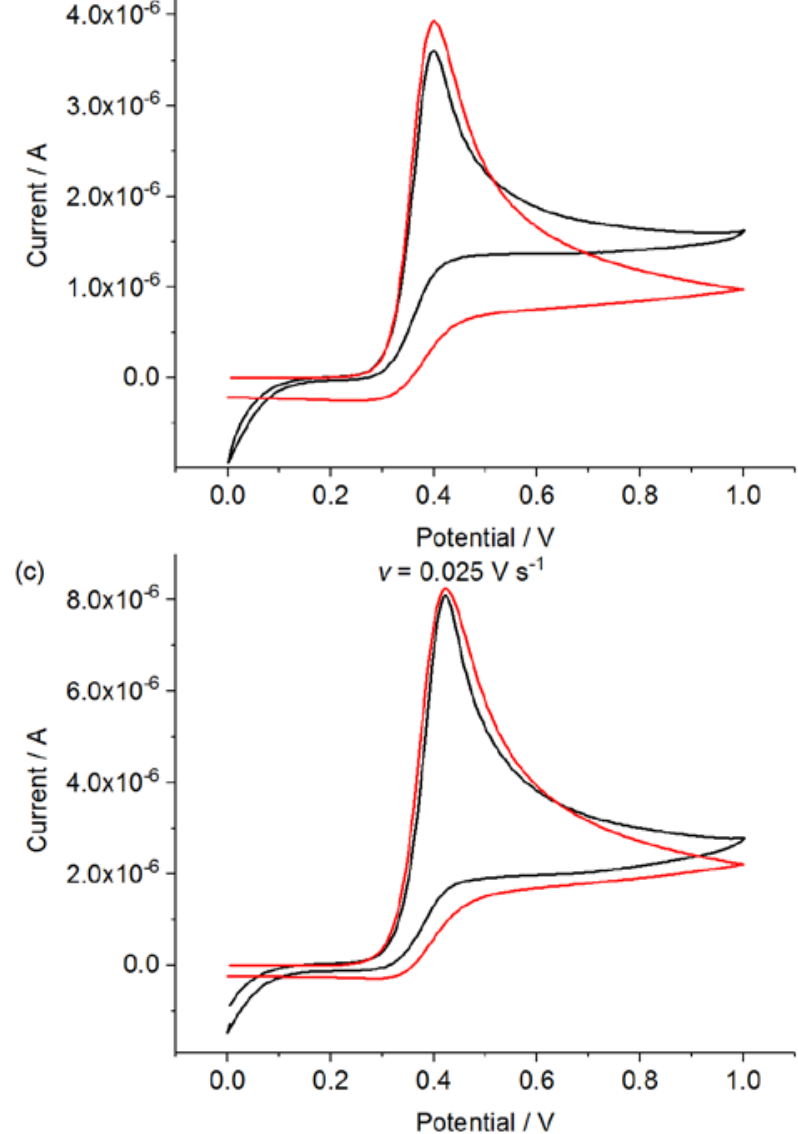

(e) $\left.2.0 \times 10^{-5}\right] \quad v=0.1 \mathrm{~V} \mathrm{~s}^{-1}$

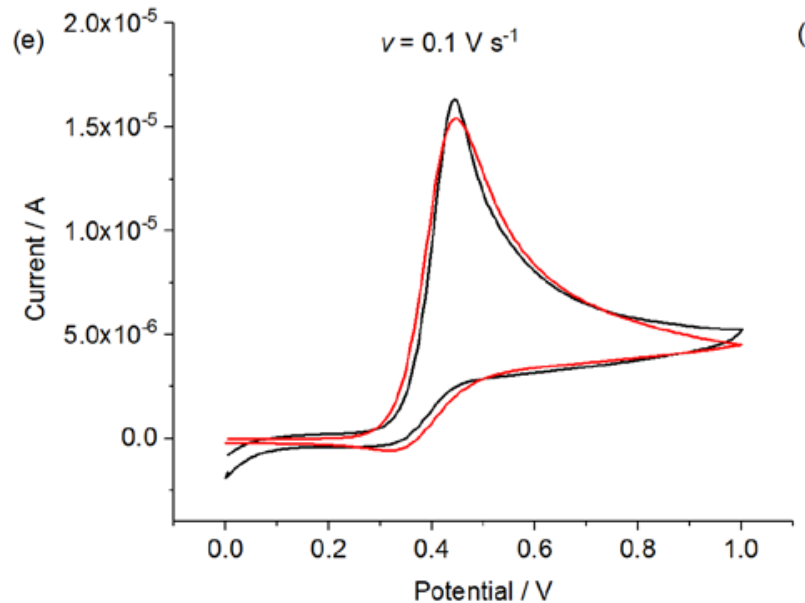

Fig. 2. CV from experiment (black) and simulation (red) at $\mathrm{s}^{-1}$, (d) $0.05 \mathrm{~V} \mathrm{~s}^{-1}$, (e) $0.1 \mathrm{~V} \mathrm{~s}^{-1}$, and (f) $0.2 \mathrm{~V} \mathrm{~s}^{-1}$.

the two waves suggestive of a split wave where the chemical step is partially completed [54].

From the above general approach the possible chemical reaction pathway can next be discussed further. It is known that the aqueous sulphite anion $\left(\mathrm{SO}_{3}{ }^{2-}\right)$ equilibrates with bisulphite and sulphur dioxide depending on the solution $\mathrm{pH}$ with the following reactions.
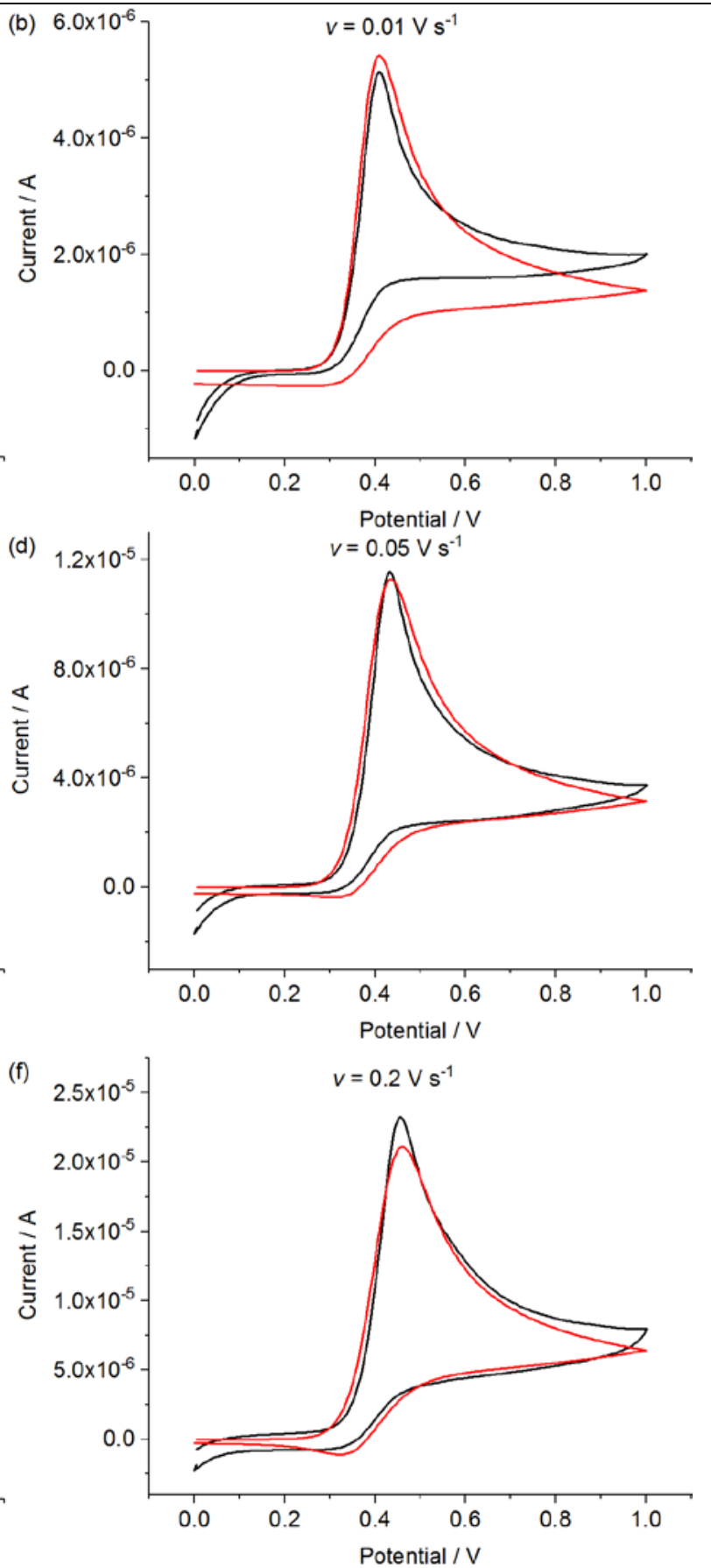

different scan rates of (a) $0.005 \mathrm{~V} \mathrm{~s}^{-1}$, (b) $0.01 \mathrm{~V} \mathrm{~s}^{-1}$, (c) $0.025 \mathrm{~V}$

$\mathrm{SO}_{2}+\mathrm{H}_{2} \mathrm{O} \rightleftharpoons \mathrm{HSO}_{3}{ }^{-}+\mathrm{H}^{+}$

$\mathrm{HSO}_{3}{ }^{-} \rightleftharpoons \mathrm{SO}_{3}{ }^{2-}+\mathrm{H}^{+}$

Although equilibrium constants reported in the literature vary, the corresponding pKa values $\left(25{ }^{\circ} \mathrm{C}\right)$ widely accepted are 1.8 and 7.2 for the above reactions, respectively [17, 55-57]. So the equilibrium concentrations of the three sulphur (IV) species can be 
determined as a function of the solution $\mathrm{pH}[17,55]$. The results in Fig. S3 show that the dominant species is aqueous sulphur dioxide $\left(\mathrm{SO}_{2}\right)$ when $\mathrm{pH}<1.85$. Bisulphite $\left(\mathrm{HSO}_{3}{ }^{-}\right.$) dominates in the range of $1.85<\mathrm{pH}$ $<7.20$ whereas sulphite $\left(\mathrm{SO}_{3}{ }^{2-}\right)$ is the major species in the solution of $\mathrm{pH}>7.20[56,58]$. Hence, $\mathrm{SO}_{2}$ is the major species during the experiments in this work since the $\mathrm{pH}$ of the supporting electrolyte of $0.10 \mathrm{M} \mathrm{H}_{2} \mathrm{SO}_{4}$ is far below 2. A previous electrochemical study [59] indicated that the oxidation of $\mathrm{SO}_{2}$ on platinum in sulphuric acid may involve a chemical step via the formation of the dithionate $\left(\mathrm{S}_{2} \mathrm{O}_{6}{ }^{2-}\right)$ accredited by spectroscopic study [60], which is consistent with the above discussion combining the fitting of $\mathrm{EC}_{2}$ model with the speciation via the following reactions: $\mathrm{SO}_{2}+\mathrm{H}_{2} \mathrm{O} \rightarrow \mathrm{H}_{2} \mathrm{SO}_{3}{ }^{+}+\mathrm{e}^{-}$

$2 \mathrm{H}_{2} \mathrm{SO}_{3}{ }^{+} \rightarrow \mathrm{H}_{2} \mathrm{~S}_{2} \mathrm{O}_{6}+2 \mathrm{H}^{+}$

Although dithionic acid $\left(\mathrm{H}_{2} \mathrm{~S}_{2} \mathrm{O}_{6}\right)$ may decompose via a disproportionation step, dilute solutions of the acid are known to be stable with the occurrence of decomposition on concentration only above $50{ }^{\circ} \mathrm{C}$ [61].

\subsection{Electrodeposition of gold particles and characterisation}

Having established an electrochemical mechanism using a gold macroelectrode, the behaviour of gold particles dispersed on supporting electrodes for electro-oxidation of sulphite was next studied. First, a facile preparation method of Au deposition onto boron doped diamond was developed. A nucleation-growth pulse protocol was applied for gold particles deposition on BDD in $1.0 \mathrm{mM}$ $\mathrm{HAuCl}_{4}$ solution. The potential and current versus time curves using deposition $\mathrm{Au}$ (150) as an example are shown in Fig. S4. A nucleation pulse was applied at $-0.7 \mathrm{~V}$ versus $\mathrm{Ag} / \mathrm{AgCl}$ for $2 \mathrm{~s}$; these parameters were chosen to enable the fast electrodeposition of gold nuclei and to avoid hydrogen evolution [45-46]. A growth pulse was applied at $0 \mathrm{~V}$ for different times and utilised to maintain a small cathodic current observed in Fig. S4(b) during the growth stage. The durations of growth pulses were changed to be $0,10,50$, and $150 \mathrm{~s}$ for different depositions. Au particle deposition onto the BDD electrode surface was confirmed by SEM analysis.

The SEM images of gold particles on the BDDs under different deposition conditions are shown in Fig. 3 at three different magnifications. It is apparent that the deposition pulse without any growth step yields quasispherical gold nanoparticles (AuNPs) as shown in Fig. 3(i) under high magnification. Traces of lower aspect ratio protrusions can be observed on branched gold nuclei structures for the $10 \mathrm{~s}$ growth pulse. The branched geometry tendency becomes more obvious for 50 s growth in Fig. 3(k) while growth deposition for 150 $\mathrm{s}$ yields star-like structures consisting of a few branches around a gold centre in Fig. 3(1). This result demonstrates that the Au particle morphology transition from quasi-spherical to star shaped can be easily controlled via the duration of the growth pulse.

A statistical analysis of particle sizes was carried out based on the SEM images of Au particles using ImageJ. To provide the information of the particle diameter, hundreds of particles of 450,698, 436, and 1279 were analysed for each deposition of $\mathrm{Au}(0), \mathrm{Au}(10), \mathrm{Au}$ (50), and $\mathrm{Au}$ (150), respectively, with the number notation in the bracket being the growth pulse time defined in the experiment section above. The size-based histograms of the gold particles were generated as shown in Fig. 3(m-p). The average diameter of Au (0) is $95 \pm 39 \mathrm{~nm}$ and $44 \%$ of them are within the range of $105-145 \mathrm{~nm}$. The size of $\mathrm{Au}(10)$ is $170 \pm 51 \mathrm{~nm}$, and $60 \%$ of the particles are $155-195 \mathrm{~nm}$. With longer growth times, the average sizes increase to be $217 \pm 80$ $\mathrm{nm}$, and $269 \pm 102 \mathrm{~nm}$ for $\mathrm{Au}$ (50) and Au (150), respectively, with the corresponding maximum percentage of $30 \%$ and $25 \%$ in the size range of 225 265 and $255-295 \mathrm{~nm}$. The results show that it is feasible to tune the particles of different size from the nanometer to the submicron scale via the growth time.

For particle arrays on a supported inert substrate, the electrochemical response of the electrode depends on the size and morphology of particles, the diffusion coefficient of analyte, $D$, and the interparticle distance (nanoparticle surface coverage), $d$, assuming that electrolysis is confined to the particles and not the support [62]. A further but key parameter is the timescale of the experiment, $t$, controlled by varying scan rate, so that if $d \gg \sqrt{D t}$, the particles are diffusional isolated but at larger times, the diffusion fields of individual particle overlap. Accordingly, the diffusional regime may be divided to five cases [63]. Case 1 corresponds to the diffusion field at isolated particles being linear at very short times where $r_{p}>$ $\sqrt{D t}\left(r_{p}\right.$ is the radius of particle). Case 2 describes the diffusion to the each particle is convergent over a longer timescale when $r_{p}<\sqrt{D t}$. In both cases of 1 and 2, adjacent particles are far enough that they are diffusionally independent on the time scale of the experiment. Case 3 is seen when the diffusional fields of neighbouring particles partially overlap where the diffusion layer thickness of each particle is comparable to the interparticle separation. When the diffusion layers of adjacent particles overlap heavily, such that the diffusion to the whole electrode surface is linear in 


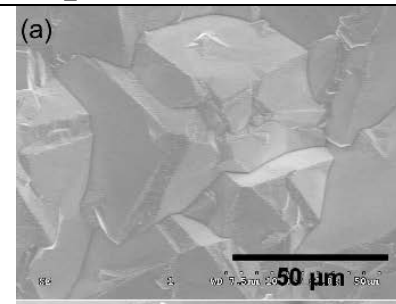

(e)
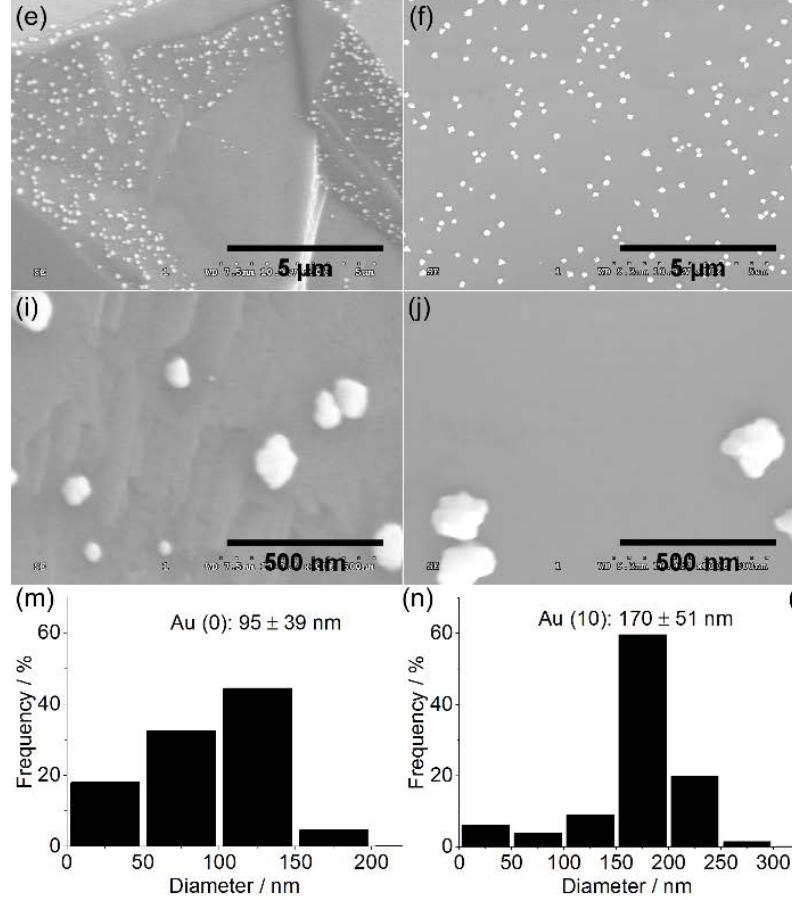

Fig. 3. SEM images of (a, e, i) Au (0), (b, f, j) Au (10), (c, g, k) deposition at three different magnifications of (a-d) $1000 \times$, (e-h) (0), (n) Au (10), (o) Au (50), and (p) Au (150) particles.

nature, it is categorised as case 4 . The above four cases are realized assuming the substrate is macroscopic in size [64-65]. In case 5, convergent diffusion to the whole substrate is achieved in the very long experimental timescale, giving sigmoidal shaped voltammograms when the substrate is of the microscale.

The images in Fig. 3(a-d) show that the particle coverage increases with longer deposition time. Next we analyse the interparticle separation in more detail. For $\mathrm{Au}(0)$, the nanoparticles are located mostly at the BDD edge areas from Fig. 3(a) although there are a few nanoparticles are on the basal planes. This may be due to the local heterogeneities in terms of differential boron dopant level [66]. The AuNPs may nucleate at the high conductivity regions where there is a higher boron concentration within the domains during the very short nucleation pulse while only a few nanoparticles are formed on the low boron-doped facets.

In order to know the interparticle distance for $\mathrm{Au}(0)$ and the resulting diffusion category correspondingly, (c) (d)

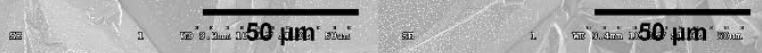

(g)
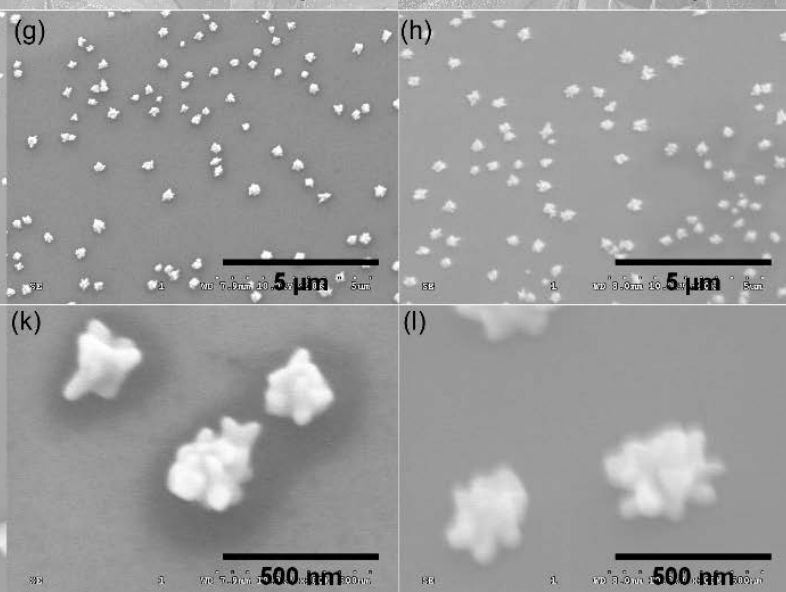

(I)
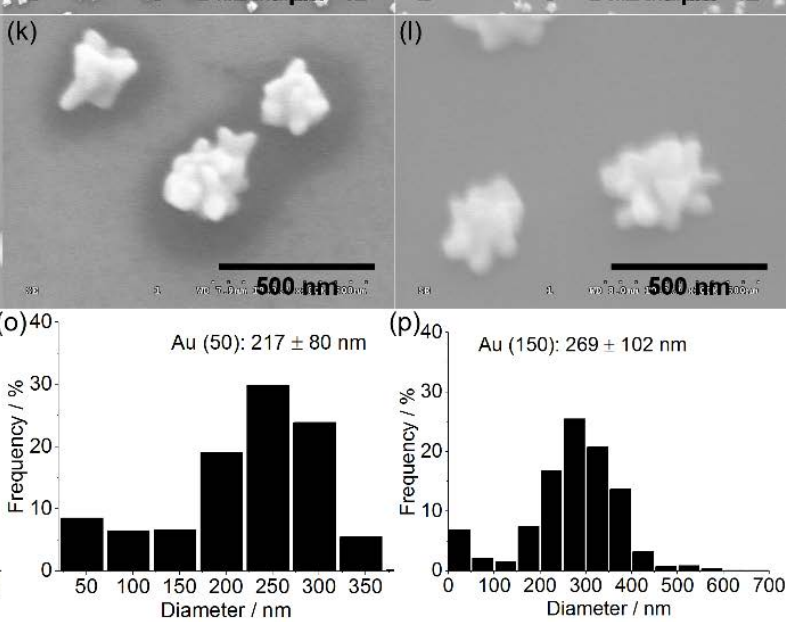

Au (50), (d, h, l) Au (150) particles after different growth times $10000 \times$, and (i-l) $100000 \times$. The size distributions of the (m) Au

we simplified the Au (0) deposition model described below via the following assumptions. First within the edge domains, the AuNPs are very close to each other and these edge domains can be simply assumed from a diffusional perspective as the individual bigger $\mathrm{Au}$ "arrays/blocks" highlighted in the Fig. S5 as irregular shapes. Simultaneously, to simplify the calculation to know the block distance, the basal planes are further assumed to be "blank" areas "without" AuNPs. Hence the entire BDD can be viewed as several randomly distributed Au arrays of flat discs of a few microns in diameter, which are separated by the "blank" BDD domains.

For Au (0), the area of each circled Au block in Fig. S5 was measured by ImageJ. Each Au block with the area $(S)$ is approximated as a disc, such that $S=\pi \mathrm{d}_{\text {eff }}{ }^{2} / 4$. Therefore the effective diameter of each Au block can be calculated (data not shown here). Then a mean diameter for these $\mathrm{Au}$ blocks can be further approximated, which is $7.9 \pm 2.9 \mu \mathrm{m}$. Assuming that the $\mathrm{Au}$ particle blocks compactly occupy the whole 
electrode in the pattern of square grid each with the same side length, the centre-to-centre distance between two neighbouring blocks is the value of the side length, which is given by:

$\sqrt{\frac{\text { area }}{\text { number of blocks }}}=16.4 \mu \mathrm{m}$.

For $\mathrm{Au}$ (10), $\mathrm{Au}$ (50) and $\mathrm{Au}$ (150), more gold particles were deposited onto the flat facets. The assumption of "blank" basal planes was not taken for these cases, as it is especially notable that gold submicron-particles are distributed quite uniformly forming a monolayer on the BDD surface in two dimensions for $\mathrm{Au}$ (150) from Fig. 3(d). Therefore the detailed interparticle distances in these basal planes circled in the Fig. S5(b-d) for these three Au deposits are analysed individually below.

To calculate the interparticle spacing it was assumed that the particles are homogeneously distributed on each of the highlighted areas for $\mathrm{Au}$ (10), $\mathrm{Au}(50)$ and $\mathrm{Au}$ (150). For these Au deposits, at least 20 areas in the each SEM were chosen randomly for analysis in order to get good statistics. The interparticle separation was calculated assuming a regular square lattice. The chosen area was assumed to be split into square cells each of equal area. Each Au particle was considered to be at the centre of a unit cell featuring an identical side length of square. Accordingly, with this approximation the centre-to-centre distance between adjacent particles was also equal to the side length as following:

the average interparticle distance $=\sqrt{\frac{\text { rectangle area }}{\text { number of particles }}}$

The rectangle area and particle statistics required for the calculation were measured and counted via the image analysis software ImageJ. So the interparticle separation was obtained via the above equation within each highlight area (data not shown here). Furthermore, the mean interparticle distance for each gold deposit is estimated by averaging the corresponding values in these analysis rectangle areas. Accordingly, with longer deposition time, the mean interparticle distances for $\mathrm{Au}$ (10), $\mathrm{Au}(50)$ and $\mathrm{Au}(150)$ are $1.43 \pm 0.26,1.19 \pm 0.12$, and $0.83 \pm 0.12 \mu \mathrm{m}$, respectively.

The results of the above discussion are summarised in the Table S2. The Au (0) blocks of micron size are separated by $16 \mu \mathrm{m}$ while for the other three gold deposits the interparticle distance is about $1 \mu \mathrm{m}$. From Table S2, the interparticle separation decreases with longer deposition time, which reflects the increasing particle density observed from Fig. 3(a-d). In essence, the system can be viewed as gold (sub)microelectrode arrays with inter-array separation of micrometre size on the BDD substrate. According to the above results, and four categories of the diffusional behaviour at a nanoparticle array depending on the particle size, interparticle distance, and $\sqrt{D t}$ (estimated as 200-1265 $\mu \mathrm{m}$ by approximating $D=4 \times 10^{-9} \mathrm{~m}^{2} \mathrm{~s}^{-1}$ and $t=10-400$ $\mathrm{s}$ via various scan rates of $0.005-0.2 \mathrm{~V} \mathrm{~s}^{-1}$ ), it is case 4 for this present study, which is the diffusion domains of neighbouring particles heavily overlap such that diffusion to the whole electrode surface is linear [62]. To this end, the current results show that the nucleationgrowth pulse deposition strategy is a facile and powerful method to construct metal particle arrays of controllable nano- and submicron- structures in terms of size and shape onto boron-doped diamond electrode surfaces, resulting in mass transport to the surface, which is planar diffusional, and hence expected to give voltammetric signals reflecting the geometric area of the BDD substrate.

\subsection{Electrochemical oxidation of sulphite on gold particle modified boron doped diamond electrodes}

Having established the successful fabrication of Au arrays on BDD substrates, the next step was to evaluate the electrochemical response of the as-prepared BDD electrode interfaces for sulphite oxidation in $\mathrm{H}_{2} \mathrm{SO}_{4}$ electrolyte solution using $\mathrm{CV}$ technique. The comparison between $\mathrm{Au}(0), \mathrm{Au}(10), \mathrm{Au}(50)$, and $\mathrm{Au}$ (150) modified BDD electrodes was first carried out in $1.0 \mathrm{mM} \mathrm{Na}_{2} \mathrm{SO}_{3}$. Simultaneously, it is also of significance to evaluate the nature of the sulphite oxidation process to distinguish between surface bound or diffusional behaviour. For this purpose a scan rate dependence study was run at the same time for each $\mathrm{Au}$ modification. Fig. 4 shows the experimentally recorded voltammograms for several $\mathrm{Au}$ particle modified BDD electrodes with variable scan rates towards the oxidation of sulphite. The peak currents of sulphite oxidation increase along with the increase of scan rates and all the curves show the peak from 0.4 to $0.6 \mathrm{~V}$ with similar waveshapes. There is a potential shift to more positive position for each gold deposit, which is in agreement with the findings using the gold macroelectrode above. The good linearity in the plot of the peak current versus the square root of scan rate is observed for all the cases, indicating that this behaviour is a diffusional controlled process instead of an absorption confined one where peak currents are proportional to the scan rate.

To attain the further insights about the electrode transfer behaviour for the sulphite oxidation process, Tafel analysis was examined again for the several gold particles modified electrodes. The Tafel plots for $\mathrm{Au}(0)$, $\mathrm{Au}$ (10), Au (50), and Au (150) modified electrodes are charted in Fig. S6-9, respectively. The 

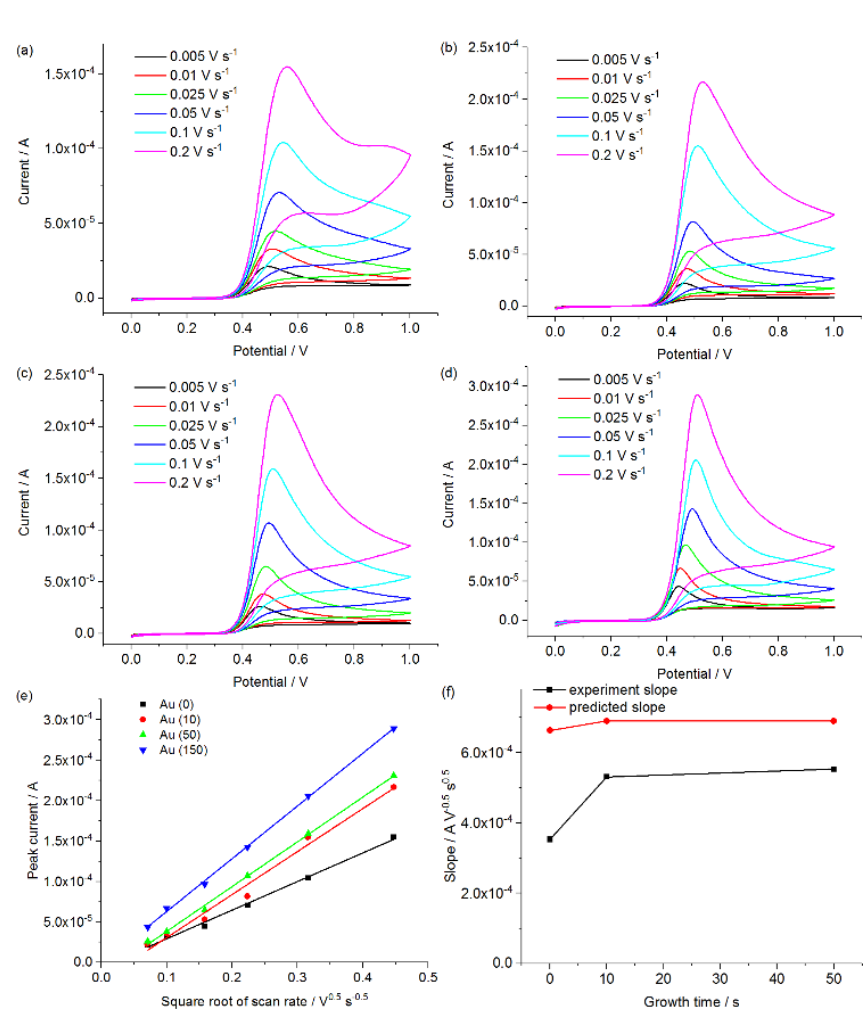

Fig. 4. CV of (a) Au (0), (b) Au (10), (c) Au (50), and (d) Au (150) particles modified BDD electrodes in $1.0 \mathrm{mM} \mathrm{Na}_{2} \mathrm{SO}_{3} / 0.10$ $\mathrm{M} \mathrm{H}_{2} \mathrm{SO}_{4}$ at different scan rates. (e) Plot of peak current as a function of the square root of scan rate from 0.005 to $0.2 \mathrm{~V} \mathrm{~s}^{-1}$ for the corresponding gold modified BDDs. (f) The comparison of experimental (black) and predicted (red) slope of the plot.

corresponding effective anodic transfer coefficient $(\beta)$ is calculated via the Tafel equation mentioned before in the Table S3-6. The results are summarized in Table S7. It shows an increasing trend for charge transfer coefficient from 0.84 to 0.95 . The $\beta$ value for Au (150) modified BDD is close to unity, indicating again a one-electron transfer electrochemically reversible process as seen for the gold macroelectrode. This corresponds to case 4 where diffusion fields of neighbouring gold particles overlap heavily for sulphite oxidation resulting in the planar diffusion toward the whole electrode. The voltammetry response is almost equivalent to that of a macroelectrode of the same geometric area as the modified electrode using much less gold particles acting as two-dimensional monolayer arrays with reducing the cost simultaneously. Hence the geometric area of 0.37 $\mathrm{cm}^{2}$ can be used for calculation of diffusion coefficient of sulphite via the Randles-Sevcik equation for electrochemically reversible process with one electron transfer involved for $\mathrm{Au}$ (150). With the plot slope (6.51 $\times 10^{-4} \mathrm{~A} \mathrm{~V}^{-0.5} \mathrm{~s}^{0.5}$ ) of the peak current versus the square root of scan rate for $\mathrm{Au}$ (150) in Fig. 4(e), the diffusion coefficient value is $4.28 \times 10^{-9} \mathrm{~m}^{2} \mathrm{~s}^{-1}$, confirming the above result achieved by a gold macroelectrode. Fig. 4(e) shows that the electrodes found by electrodeposition for $0,10,50$ s second lower gradients in the Randles-Sevcik plot of $I_{p}$ versus $v^{0.5}$ as compared to $\mathrm{Au}$ (150) with the slopes being the evaluation of electrochemical reversibility. This reflects an increased level of electrochemical irreversibility as the deposition time shortens:

$I_{p}=2.99 \times 10^{5} A D^{0.5} C v^{0.5} n\left(n^{\prime}+\beta\right)^{0.5}$

where the above physical quantities have their general meanings as stated before except that $n^{\prime}$ is the number of electrons transferred before the rate determining step. This in turn suggests that the morphology of the nanoparticles influences the electron transfer rate. Moreover, the slope of $I_{p}$ versus $v^{0.5}$ was predicted using $D=4.28 \times 10^{-9} \mathrm{~m}^{2} \mathrm{~s}^{-1}, n^{\prime}=0$, and the measured $\beta$ values in Table S7. Although the interparticle spacing is as estimated above for $\mathrm{Au}(0)$, Au (10), and Au (50), small compared to diffusion layer thickness associated with the voltammetry at the particle, it should be noticed that the prediction of peak currents using equation (10) is inappropriate for the present situation since the process at each particle involves following second order kinetics. As we move "up" the wave towards the peak current the following reaction occurs to a greater extent as the reaction is second order. Accordingly, the extent of "chemically promoted electrochemical irreversibility" at the peak current drops below that estimated from equation (10) in Fig. 4 [67].

As noted above that the Au (150) modified electrode configuration and electrochemical response, and the recognition that it behaves on a gold macroelectrode, a sulphite concentration dependence was evaluated. Different concentrations of sulphite were chosen for $\mathrm{Au}$ (150) particle modified BDD at 10, 20, $200 \mu \mathrm{M}, 1.0$ $\mathrm{mM}$, and $2.0 \mathrm{mM}$ at a fixed scan rate of $0.05 \mathrm{~V} \mathrm{~s}^{-1}$ as shown in Fig. 5. At high concentrations of $1.0 \mathrm{mM}$ and $2.0 \mathrm{mM}$, there is only a forward oxidation peak without back peaks. A very tiny reversal peak rises with sulphite being a moderate concentration of $200 \mu \mathrm{M}$. With decreasing the concentration to 20 and $10 \mu \mathrm{M}$ further, there are obvious backward peaks at $0.38 \mathrm{~V}$, indicating that the presence of back peaks is relevant to the sulphite concentration. As mentioned before that $\mathrm{EC}_{2}$ scheme has a dependence of the bulk concentration of analyte, these results using gold particle modified electrode show similar electrochemical oxidation behaviour with that on the gold macroelectrode, further providing evidence for the $\mathrm{EC}_{2}$ hypothesis. Furthermore, Fig. 5(f) 
shows that the anodic peak currents increase linearly with the concentration of sulphite with the slope value of $1.4 \times 10^{-4} \mathrm{~A} \mathrm{~mol}-1 \mathrm{~m}^{3}$, with which the electrochemically reversible Randles-Sevcik equation was applied at a scan rate of $0.05 \mathrm{~V} \mathrm{~s}^{-1}$ for achieving the diffusion coefficient value of sulphite of $3.8 \times 10^{-9} \mathrm{~m}^{2} \mathrm{~s}^{-}$ ${ }^{1}$. This is also consistent with the above values via the linear dependence of peak current versus square root of scan rate on both gold macroelectrode and BDD decorated by $\mathrm{Au}(150)$ at $1.0 \mathrm{mM}$ sulphite.
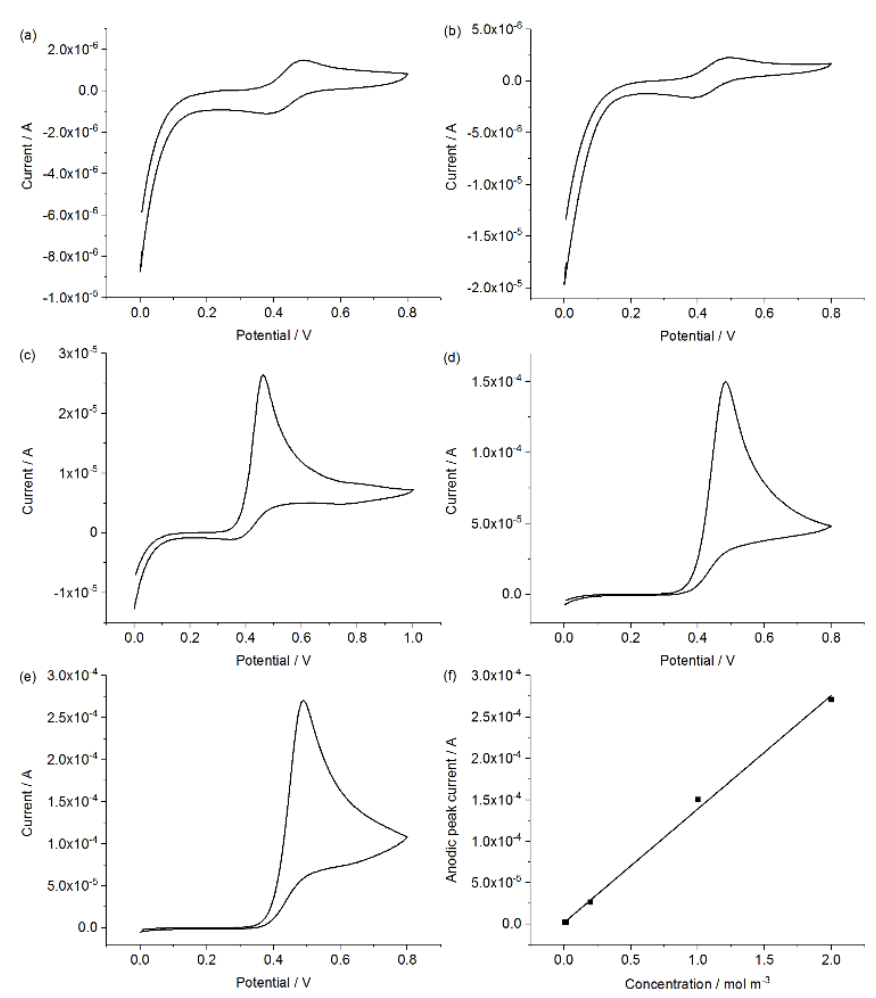

Fig. 5. CV of sulphite at different concentrations of (a) $10 \mu \mathrm{M}$, (b) $20 \mu \mathrm{M}$, (c) $200 \mu \mathrm{M}$, (d) $1.0 \mathrm{mM}$, and (e) $2.0 \mathrm{mM}$ at the $\mathrm{Au}$ (150)-BDD electrode at $0.05 \mathrm{~V} \mathrm{~s}^{-1}$. (f) Plot of anodic peak currents versus the concentration of sulphite.

Next the simulation was carried out again to examine the above model across the concentration range. The fitting parameters were as follows: $E^{0}=0.44 \mathrm{~V}, \alpha=0.5$, $k_{s}=0.007 \mathrm{~cm} \mathrm{~s}^{-1}, K_{e q}=1 \times 10^{6}, k_{f}=1 \times 10^{4} \mathrm{M}^{-1} \mathrm{~s}^{-1}, D_{A}=$ $D_{B}=D_{C}=4 \times 10^{-9} \mathrm{~m}^{2} \mathrm{~s}^{-1}, v=0.05 \mathrm{~V} \mathrm{~s}^{-1}$, the equivalent geometric area $A=3.7 \times 10^{-5} \mathrm{~m}^{2}$ of Au particles in terms of planar diffusional conception. The kinetic parameters for the second order chemical step are the same as those of gold macroelectrode while the kinetics of the electrochemical step are close to those of gold substrate. The overlaid voltammograms of experiment and simulation are shown at $10 \mu \mathrm{M}$ and $2.0 \mathrm{mM}$ of sulphite as examples in Fig. 6 while the other concentration fittings between these two are present in the Fig. S10. The simulation fits well across the full range of concentrations differing by more than two orders of magnitude regarding the voltammetric shapes with peak currents and potentials. Note that the region below 0.2 $\mathrm{V}$ may additionally reflect double-layer charging capacitance behaviour since large gold particles are decorated onto the BDD substrate likely overcoming its own low background capacitance.
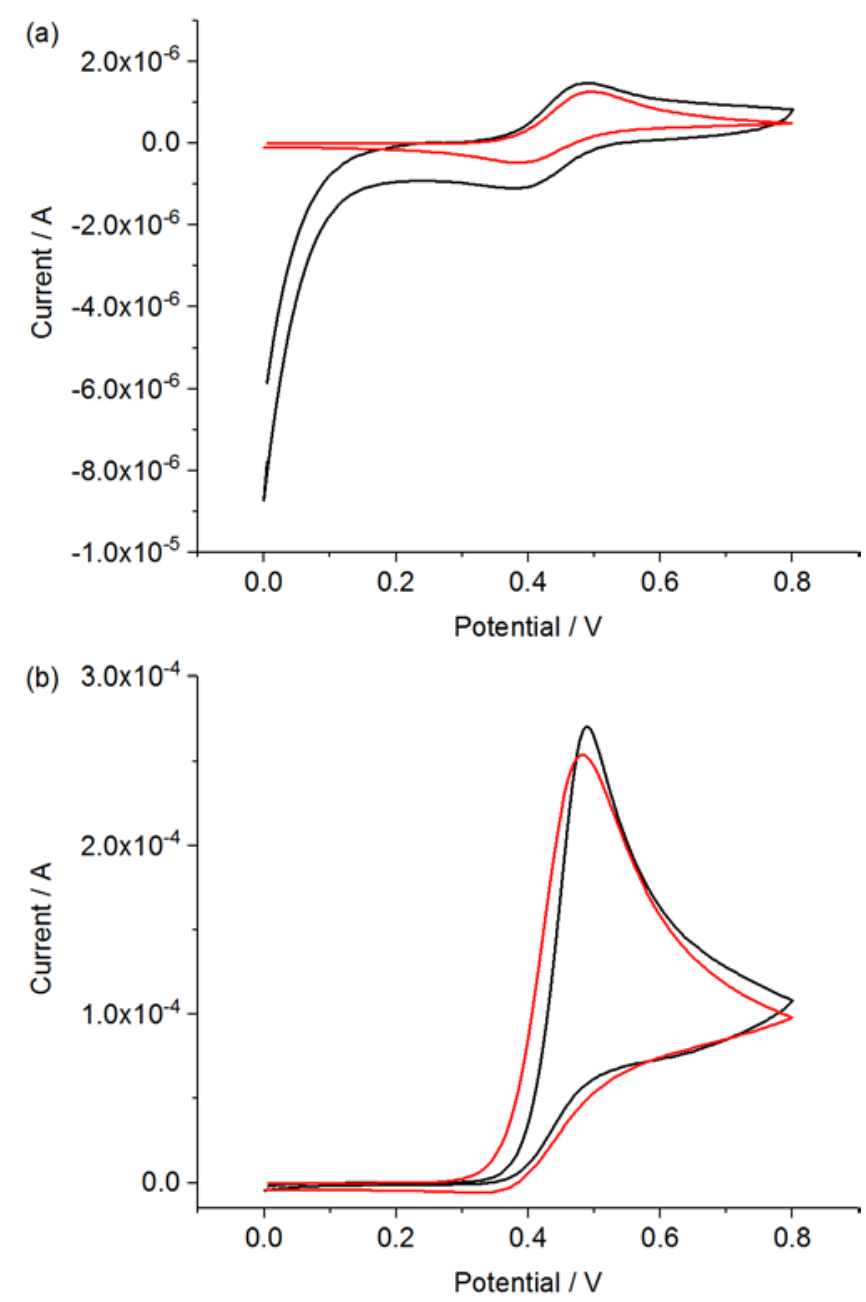

Fig. 6. CV from experiment (black) and simulation (red) at different sulphite concentrations of (a) $10 \mu \mathrm{M}$, and (b) $2.0 \mathrm{mM}$ at the $\mathrm{Au}$ (150)-BDD electrode at a scan rate of $0.05 \mathrm{~V} \mathrm{~s}^{-1}$.

The transport characteristics of the electrode were investigated with different scan rates. Two extremes are considered; one is at a high concentration of $2.0 \mathrm{mM}$ and the other is at a low concentration of $10 \mu \mathrm{M}$. Fig. 7 displays $\mathrm{CV}$ data of the sulphite redox process varying scan rates of $0.005-0.05 \mathrm{~V} \mathrm{~s}^{-1}$. At $10 \mu \mathrm{M}$, reverse peaks show at $0.38 \mathrm{~V}$ while no such peaks at $2.0 \mathrm{mM}$, indicating that an enhanced concentration promotes the 
follow-up chemical kinetics and therefore, the loss of the reverse peak within the $\mathrm{EC}_{2}$ scheme. Besides, not only the anodic but also cathodic peak currents have a linear fitting as a function of square root of scan rate in $10 \mu \mathrm{M}$ sulphite in Fig. 7(b), suggesting the sulphite redox process is diffusion limited. So does the anodic process in the high concentration of $2.0 \mathrm{mM}$ sulphite.
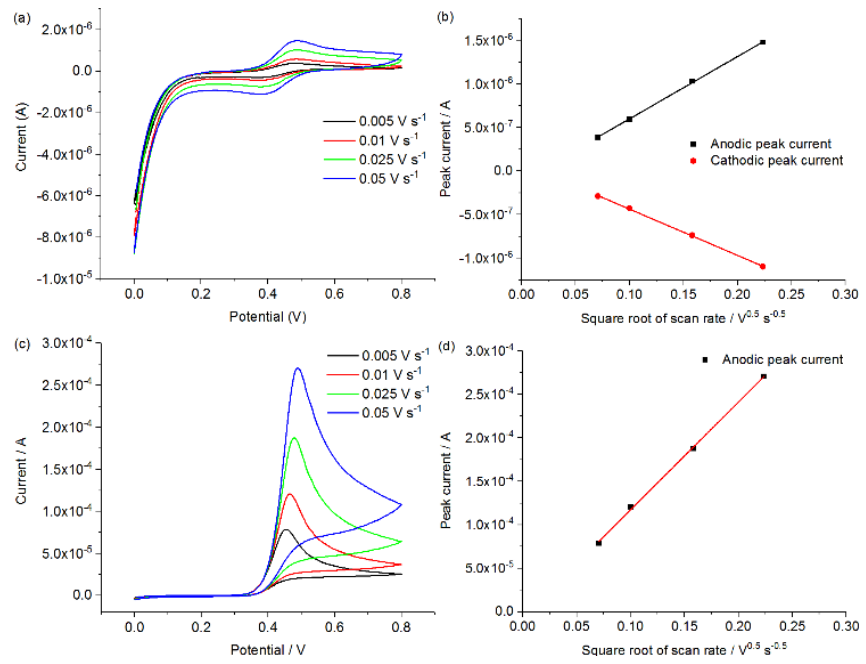

Fig. 7. CV of sulphite in the concentrations of (a) $10 \mu \mathrm{M}$, and (c) $2.0 \mathrm{mM}$ at different scan rates of $0.005-0.05 \mathrm{~V} \mathrm{~s}^{-1}$ at $\mathrm{Au}$ (150)BDD electrode. Plot of anodic and cathodic peak currents versus the square root of scan rate in (b) $10 \mu \mathrm{M}$, and (d) $2.0 \mathrm{mM}$ sulphite.

The $\mathrm{EC}_{2}$ model was employed again for simulation using the same parameters of $\mathrm{Au}$ (150) modified BDD. Scan rates ranging from 0.005 to $0.05 \mathrm{~V} \mathrm{~s}^{-1}$ were fitted. Fig. 8 shows the overlaid experimental and simulated voltammograms for two extreme concentrations of 10 $\mu \mathrm{M}$ and $2.0 \mathrm{mM}$ sulphite. The fitting is good in the peak potential and current across both concentrates and scan rates. The reasonable fitting (particularly for the oxidation process) over these wide ranges of both matches the general trend in the experimental CVs, and supports the indication of a one-electron oxidation process with a follow-up dimerization reaction $\left(\mathrm{EC}_{2}\right.$ mechanism). Note that the improved data in terms of signal to noise between the gold macroelectrode and the $\mathrm{Au}$ particle decorated BDD reflects the lower capacitance of the latter. This diffusional responses relating to gold macroelectrodes can be attained for the heavily decorated BDD electrodes but with a reduced capacitance facilitating modelling which is challenging on the macroelectrode.
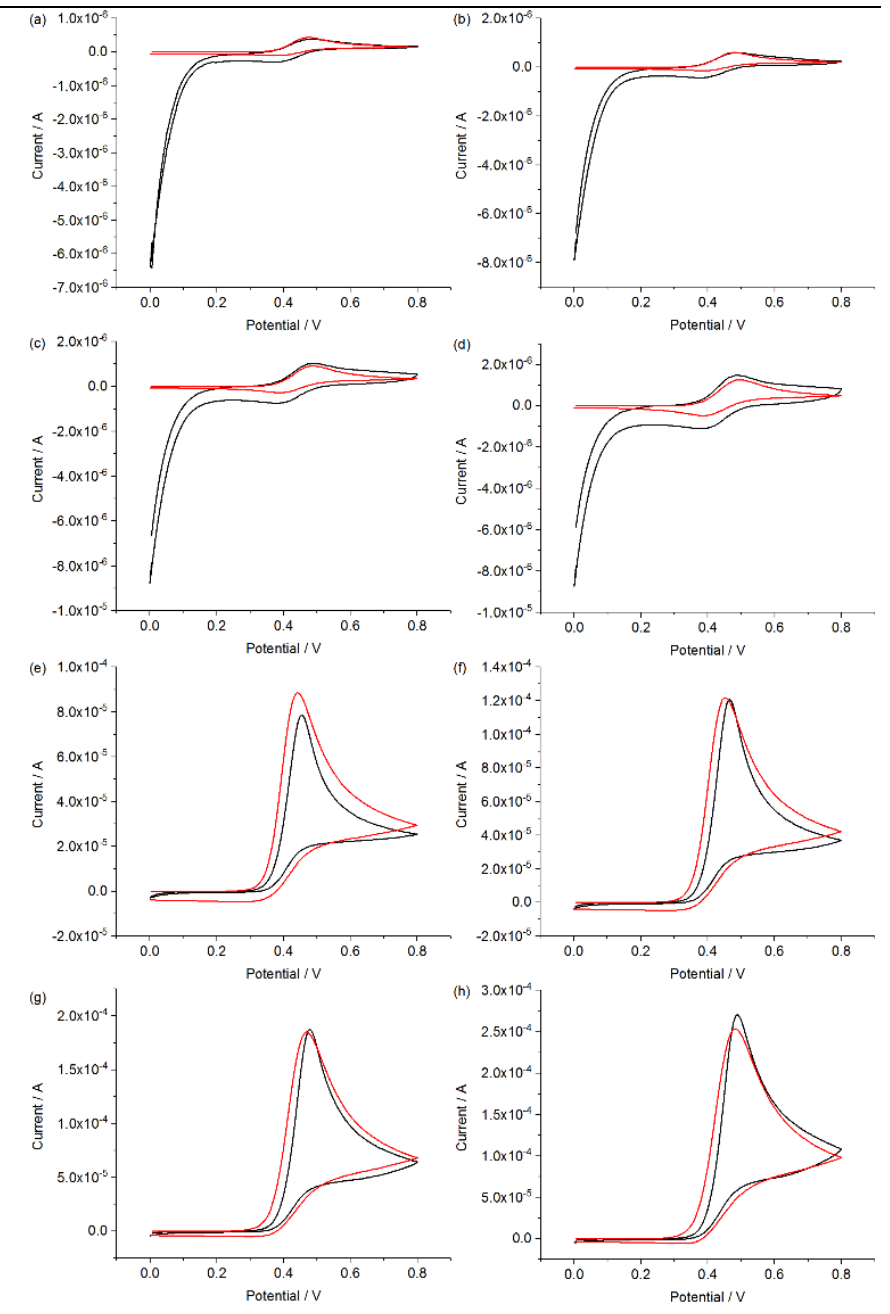

Fig. 8. The comparison of experimental (black) and simulated (red) voltammograms for the oxidation of (a-d) $10 \mu \mathrm{M}$, and (eh) $2.0 \mathrm{mM}$ sulphite at scan rates of (a, e) $0.005 \mathrm{~V} \mathrm{~s}^{-1}$, (b, f) 0.01 $\mathrm{V} \mathrm{s}^{-1}$, (c, g) $0.025 \mathrm{~V} \mathrm{~s}^{-1}$, and (d, h) $0.05 \mathrm{~V} \mathrm{~s}^{-1}$ at the Au (150)BDD electrode.

\section{Conclusions}

The electro-oxidation of sulphite has been investigated in sulphuric acid solution on Au macroelectrode and particles modified BDDs. Electrodeposition of gold particles was realized via a nucleation-growth pulse strategy. The approach enables the fabrication of gold of various sizes from nanometer to submicron-scale, and different morphologies of quasi-spherical and star-shape, with varying surface coverages. The sulphite oxidation proceeds via a one-electron transfer process followed by a second order chemical reaction, $\mathrm{EC}_{2}$ mechanism, which was supported by the interpretation of the experiment and digital simulation for both macroscopic gold electrode and the as-prepared gold particle modified BDDs. We confirm that the AuNPs deposited electrode is a good alternative for the $\mathrm{Au}$ macroelectrode for sulphite oxidation. The 
knowledge of the electro-oxidation mechanism of sulphite in acid media is of significance for various applications when using electrochemical methods to monitor sulphite wherever present such as in the food industry.

\section{References}

[1] J. P. Winiarski, M. R. de Barros, H. A. Magosso, C. L. Jost, Electrochim Acta 2017, 251, 522-531.

[2] S. Satienperakul, P. Phongdong, S. Liawruangrath, Food Chem 2010, 121, 893-898.

[3] T. Garcia, E. Casero, E. Lorenzo, F. Pariente, Sensor Actuat B-Chem 2005, 106, 803-809.

[4] V. S. Manikandan, B. Adhikari, A. C. Chen, Analyst 2018, 143, 4537-4554.

[5] S. Glasstone, A. Hickling, Journal of the Chemical Society 1933, 829-836.

[6] K. I. Rozenthal, V. I. Veselovsky, Zh. Fiz. Khim. 1953, 27, 1163.

[7] C. Quijada, A. Rodes, J. L. Vazquez, J. M. Perez, A. Aldaz, J Electroanal Chem 1995, 394, 217-227.

[8] C. Quijada, A. Rodes, J. L. Vazquez, J. M. Perez, A. Aldaz, J Electroanal Chem 1995, 398, 105-115.

[9] C. Quijada, J. L. Vazquez, A. Aldaz, J Electroanal Chem 1996, 414, 229-233.

[10] J. A. O'Brien, J. T. Hinkley, S. W. Donne, J Electrochem Soc 2010, 157, F111-F115.

[11] E. T. Seo, D. T. Sawyer, Electrochim Acta 1965, 10, 239-252.

[12] Z. Samec, J. Weber, Electrochim Acta 1975, 20, 403412.

[13] K. Varga, P. Baradlai, A. Vertes, Electrochim Acta 1997, 42, 1143-1155.

[14] C. Quijada, E. Morallon, J. L. Vazquez, L. E. A. Berlouis, Electrochim Acta 2000, 46, 651-659.

[15] Y. V. Tolmachev, D. A. Scherson, Electrochim Acta 2004, 49, 1315-1319.

[16] J. A. O'Brien, J. T. Hinkley, S. W. Donne, J Electrochem Soc 2012, 159, F585-F593.

[17] I. Streeter, A. J. Wain, J. Davis, R. G. Compton, J Phys Chem B 2005, 109, 18500-18506.

[18] A. V. Dribinskii, M. R. Tarasevich, V. E. Kazarinov, Mater Chem Phys 1989, 22, 377-400.

[19] N. A. Urisson, G. V. Schteinberg, M. R. Tarasevich, V. S. Bagotsky, N. M. Zagudaeva, Electrokhimiya 1983, 19, 275-281.

[20] T. Hunger, F. Lapicque, A. Storck, J Appl Electrochem 1991, 21, 588-596.
[21] T. Hunger, F. Lapicque, Electrochim Acta 1991, 36, 1073-1082.

[22] J. Lu, D. B. Dreisinger, W. C. Cooper, J Appl Electrochem 1999, 29, 1161-1170.

[23] C. Quijada, J. L. Vazquez, Electrochim Acta 2005, 50, 5449-5457.

[24] C. Mu, M. Hou, Y. Xiao, H. J. Zhang, S. J. Hong, Z. G. Shao, Electrochim Acta 2015, 171, 29-34.

[25] C. A. S. Brevett, D. C. Johnson, J Electrochem Soc 1992, 139, 1314-1319.

[26] K. Scott, W. M. Taama, Electrochim Acta 1999, 44, 3421-3427.

[27] P. W. T. Lu, R. L. Ammon, J Electrochem Soc 1980 127, 2610-2616.

[28] C. Quijada, J. L. Vazquez, Recent Res. Dev. Electrochem. 2000, 3, 137-181.

[29] S. Z. Chu, H. Kawamura, M. Mori, Electrochim Acta 2007, 53, 92-99.

[30] K. E. Toghill, R. G. Compton, Electroanal 2010, 22, 1947-1956.

[31] N. J. Yang, S. Y. Yu, J. V. Macpherson, Y. Einaga, H. Y. Zhao, G. H. Zhao, G. M. Swain, X. Jiang, Chem Soc Rev 2019, 48, 157-204.

[32] K. Muzyka, J. R. Sun, T. H. Fereja, Y. X. Lan, W. Zhang, G. B. Xu, Anal Methods 2019, 11, 397-414.

[33] S. J. Cobb, Z. J. Ayres, J. V. Macpherson, Annual Review of Analytical Chemistry 2018, 11, 463-484.

[34] F. W. Campbell, R. G. Compton, Anal Bioanal Chem 2010, 396, 241-259.

[35] C. M. Welch, R. G. Compton, Anal Bioanal Chem 2006, 384, 601-619.

[36] C. R. Raj, T. Okajima, T. Ohsaka, J Electroanal Chem 2003, 543, 127-133.

[37] E. Katz, I. Willner, J. Wang, Electroanal 2004, 16, 1944.

[38] M. Kizling, M. Dzwonek, A. Wieckowska, R. Bilewicz, Curr Opin Electroche 2018, 12, 113-120.

[39] Y. Y. Peng, D. Guo, W. Ma, Y. T. Long, Chemelectrochem 2018, 5, 2982-2985.

[40] J. J. Jiang, W. J. Fan, X. Z. Du, Biosens Bioelectron 2014, 51, 343-348.

[41] K. Wiesener, Electrochim Acta 1973, 18, 185-189.

[42] S. E. Lyke, S. H. Langer, J Electrochem Soc 1991, 138, 1682-1687.

[43] M. R. Tarasevich, A. V. Dribinskii, S. A. Shmakov, V. A. Rylov, Soviet Electrochemistry 1986, 22, 911-915.

[44] X. S. Cai, E. E. L. Tanner, C. H. Lin, K. Ngamchuea, J. S. Foord, R. G. Compton, Phys Chem Chem Phys 2018, 20, 1608-1614. 
[45] J. Izquierdo, B. Mizaikoff, C. Kranz, Phys Status Solidi A 2016, 213, 2056-2062.

[46] F. Bottari, K. De Wael, J Electroanal Chem 2017, 801, 521-526.

[47] M. Rudolph, D. P. Reddy, S. W. Feldberg, Anal Chem 1994, 66, A589-A600.

[48] D. L. Li, C. H. Lin, C. Batchelor-McAuley, L. F. Chen, R. G. Compton, J Electroanal Chem 2018, 826, 117-124.

[49] W. J. Albery, Electrode kinetics, Clarendon Press, Oxford, 1975.

[50] R. G. Compton, C. E. Banks, Understanding voltammetry, Third ed., World Scientific Publishing, London, 2018.

[51] D. Pletcher, R. Greff, R. Peat, L. M. Peter, J. Robinson, Instrumental Methods in Electrochemistry, Woodhead Publishing Limited, Cambridge, 2011.

[52] A. C. Testa, W. H. Reinmuth, Anal Chem 1961, 33, $1320-1324$

[53] C. Kang, J. Lee, D. S. Silvester, J Phys Chem C 2016, 120, 10997-11005.

[54] W K. R. Ward, N. S. Lawrence, R. S. Hartshorne, R. G. Compton, J Phys Chem C 2011, 115, 11204-11215.

[55] M. A. Siddiqi, J. Krissmann, P. PetersGerth, M. Luckas, K. Lucas, J Chem Thermodyn 1996, 28, 685-700.

[56] J. A. O'Brien, J. T. Hinkley, S. W. Donne, S. E. Lindquist, Electrochim Acta 2010, 55, 573-591.
[57] C. Iwasaki, T. Ohsaka, F. Kitamura, ECS Transactions 2009, 25, 671-679.

[58] A. G. Zelinsky, B. Y. Pirogov, Electrochim Acta 2017, 231, 371-378.

[59] A. J. Appleby, B. Pichon, J Electroanal Chem 1979, 95, 59-71.

[60] C. Korzeniewski, W. McKenna, S. Pons, Journal of Electroanalytical Chemistry and Interfacial Electrochemistry 1987, 235, 361-368.

[61] M. Schmidt, W. Siebert, in Comprehensive inorganic chemistry, Vol. 2, First ed. (Eds.: J. C. Bailar, H. J. Emeléus, R. Nyholm, A. F. Trotman-Dickenson), Pergamon Press, Oxford, 1973.

[62] C. Batchelor-McAuley, E. Katelhon, E. O. Barnes, R. G. Compton, E. Laborda, A. Molina, Chemistryopen 2015, 4, 224-260.

[63] C. Lin, R. G. Compton, Curr Opin Electroche 2018 https://doi.org/10.1016/j.coelec.2018.08.001

[64] T. J. Davies, S. Ward-Jones, C. E. Banks, J. del Campo, R. Mas, F. X. Munoz, R. G. Compton, J Electroanal Chem 2005, 585, 51-62.

[65] T. J. Davies, R. G. Compton, J Electroanal Chem 2005, $585,63-82$

[66] N. R. Wilson, S. L. Clewes, M. E. Newton, P. R. Unwin, J. V. Macpherson, J Phys Chem B 2006, 110, 56395646.

[67] I. Streeter, S. F. Jenkinson, G. W. J. Fleet, R. G. Compton, J Electroanal Chem 2007, 600, 285-293. 


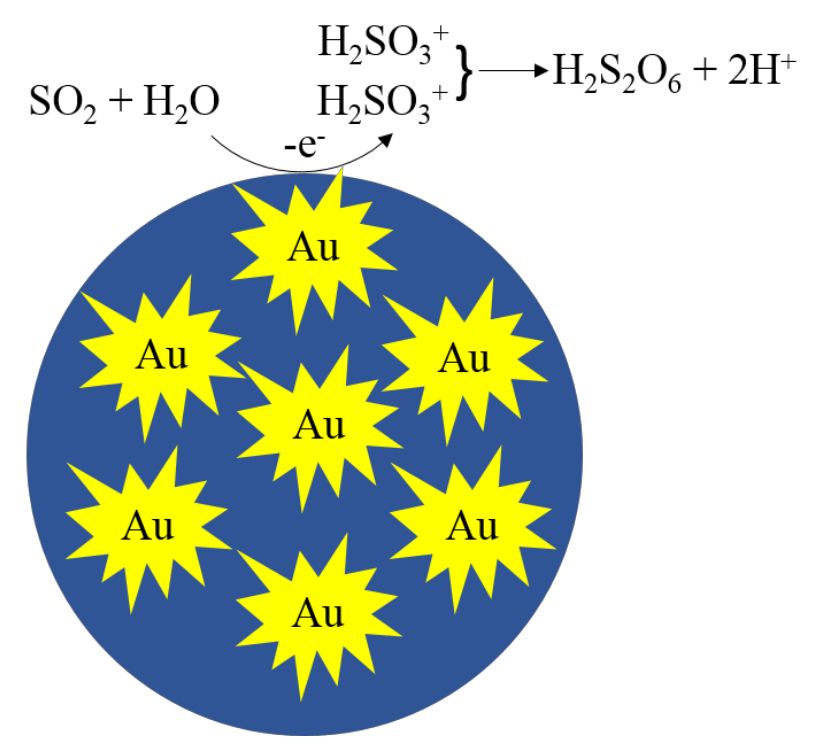

